\title{
Reconstruction of a Complex Scalp Defect after the Failure of Free Flaps: Changing Plans and Strategy
}

\author{
Youn Hwan Kim ${ }^{1}$, \\ Gyeong Hoe Kim², \\ Sang Wha Kim² \\ ${ }^{1}$ Department of Plastic and Reconstructive \\ Surgery, Hanyang University College of \\ Medicine, Seoul; \\ ${ }^{2}$ Department of Plastic and Reconstructive \\ Surgery, Seoul National University Hospital, \\ Seoul, Korea
}

No potential conflict of interest relevant to this article was reported.

\begin{abstract}
The ideal scalp reconstruction involves closure of the defect with similar hair-bearing local tissue in a single step. Various reconstructions can be used including primary closure, secondary healing, skin grafts, local flaps, and microvascular tissue transfer. A 53-year-old female patient suffered glioblastoma, which had recurred for the second time. The neurosurgeons performed radial debridement and an additional resection of the tumor, followed by reconstruction using a serratus anterior muscle flap with a split-thickness skin graft. Unfortunately, the flap became completely useless and a bilateral rotation flap was used to cover the defect. Two month later, seroma with infection was found due to recurrence of the tumor. Additional surgery was performed using multiple perforator based island flap. The patient was discharged two weeks after surgery without any complications, but two months later, the patient died. Radical surgical resection of tumor is the most important curative option, followed by functional and aesthetic reconstruction. We describe a patient with a highly malignant tumor that required multiple resections and subsequent reconstruction. Repeated recurrences of the tumor led to the failure of reconstruction and our strategy inevitably changed, from reconstruction to palliative treatment involving fast and stable wound closure for the patient's comfort.
\end{abstract}

Keywords: Perforator flap / Reconstructive surgical procedures / Surgical flaps / Scalp

\section{INTRODUCTION}

The scalp consists of specialized tissue composed of dense hair follicles and inelastic, thick galea aponeurotica, unlike other tissues of the body [1]. Reconstruction of the scalp can be challenging because of the convexity of the underlying skeleton, the inelasticity of the galea, and the paucity of the adjacent tissue, which make even small defects difficult to close [2-4]. The ideal scalp reconstruction involves closure of the defect with similar hair-bearing tissue and re-creation of the hairline [2,3]. A variety of reconstructions can be used. Primary closure should always be considered first, although it is limited by size [2,3]. Local flaps

Correspondence: Sang Wha Kim

Department of Plastic and Reconstructive Surgery, Seoul National University Hospital, Seoul National University College of Medicine, 101 Daehak-ro, Jongno-gu, Seoul 03080 , Korea

E-mail: sw1215@snu.ac.kr

Received August 6, 2016 / Revised May 30, 2017 / Accepted May 31, 2017 have the advantage of using skin adjacent to the defect and so providing the best match in color and texture [1]. With appropriate planning, excellent outcomes may be achieved. Advancement flaps, rotation flaps, transposition flaps and rhomboid flaps are used $[1,3,5]$. Finally, microvascular tissue transfer can be used [2]. This has become the best option for reconstruction of large scalp defect, especially when there is a need to cover neurocranial structures, alloplastic material, or areas with subclinical infection [2].

However, reconstruction after oncologic resection has different goals. Radical resection is the most important, followed by functional and aesthetic considerations [6]. We describe a patient with a highly malignant tumor that required multiple resections and reconstruction.

\section{CASE REPORT}

A 53-year-old female patient had a chronic headache for 5 years 
before visiting our hospital. Based on the initial magnetic resonance imaging before surgery, anaplastic astrocytoma of the left cerebellum was suspected. Neurosurgeons performed a radical resection of the tumor, and the finial pathologic diagnosis confirmed glioblastoma. The patient received radiation therapy and chemotherapy after the operation. One year later, recurrence of the tumor was suspected, and a neurosurgeon performed a second radical resection of the tumor along with cranioplasty. Four months after the operation, the wound was disrupted due to infection. A neurosurgeon removed the cranial bone and consulted our department in connection with infection control and obliteration of the dead space. Physical examination revealed a closed wound about $8 \mathrm{~cm}$ in length, but underneath the closed skin cranial bone or soft tissue was missing. Culturing led to the growth of methicillin-resistant Staphylococcus aureus. Unfortunately, tumor recurrence was also suspicious, and therefore, the neurosurgeon planned radical debridement and additional resection of the tumor, which required reconstruction. The defect was $8 \times 5 \mathrm{~cm}$ in size, with exposure of the dura mater (Fig. 1). We used a serratus anterior muscle flap with a split-thickness skin graft to cover the defect. Anastomosis was performed to superficial temporal artery and vein (Fig. 2). Even though we had also considered local flap for reconstruction, the large size and deep depth of the defect, possibility of subclinical infection and radiation injury to adjacent tissue led us to perform free flap reconstruction firstly. Unfortunately, the flap became completely useless 7 days after the operation due to hematoma and venous thrombosis. It was detached and a bilateral rotation flap used to cover the defect with grafted donor site skin (Figs. 3, 4). The wound healed completely without infection and the patient was discharged.

Two month later, the patient revisited our hospital with wound disruption and purulent discharge from the wound. Seroma with infection was found due to recurrence of the tumor (Fig. 5). Additional surgery was planned. For exposure, a rotation flap was elevated from the occipital area to create a window for the neurosurgeon to perform radical debridement and resection of the tumor. After resection, the resulting defect was approximately $8 \times 8 \mathrm{~cm}$ with exposed brain structures and could not be covered with the previous flap. To reconstruct the defect, two perforator-based flaps were designed, and a handheld Doppler was used to map the perforators adjacent to the defect. To cover the lower portion of the defect, an $8 \times 5 \mathrm{~cm}$ occipital artery perforator-based island flap

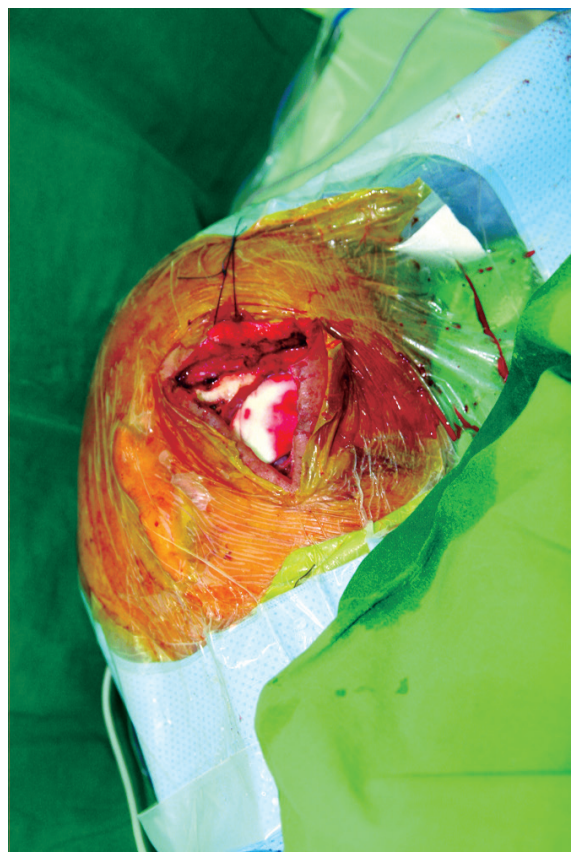

Fig. 1. Radical debridement of dead bone and infected tissue and resection of tumor resulted in dead space at posterior auricular area.

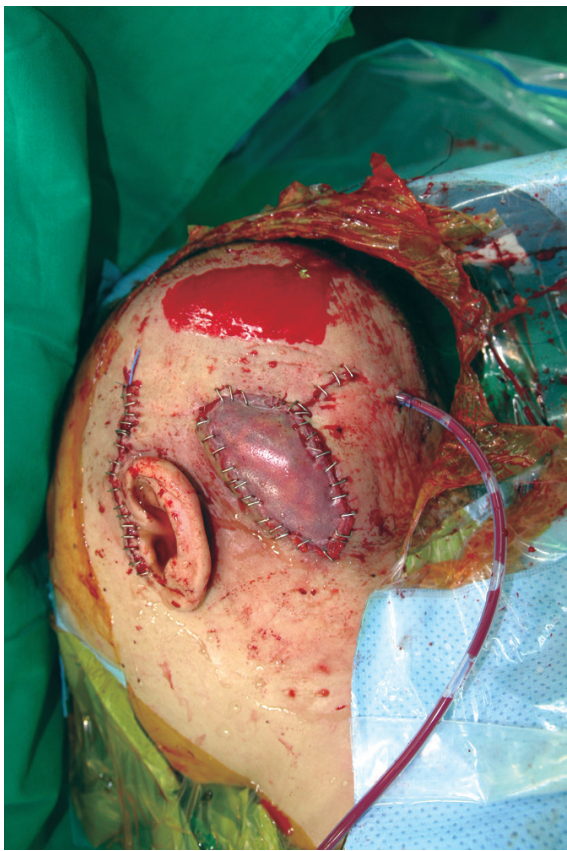

Fig. 2. Immediate postoperative view shows well-vascularized flap. 


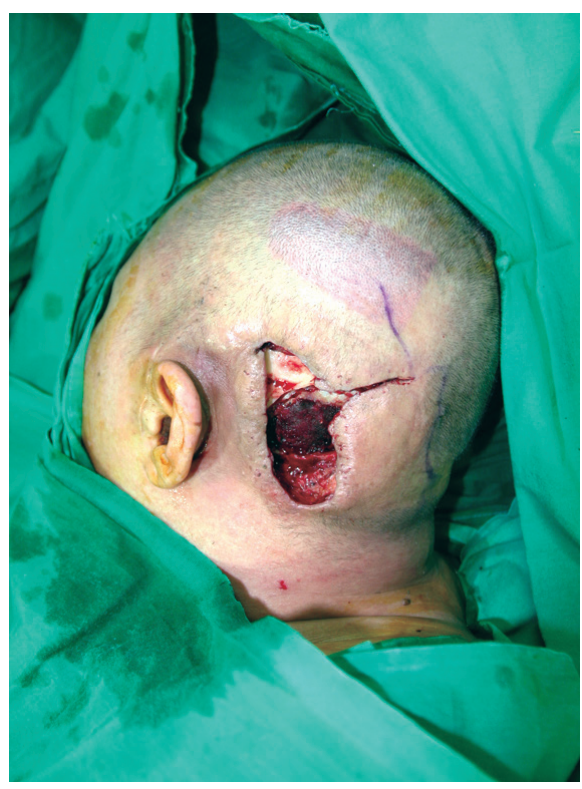

Fig. 3. Flap was detached and irrigation was performed.

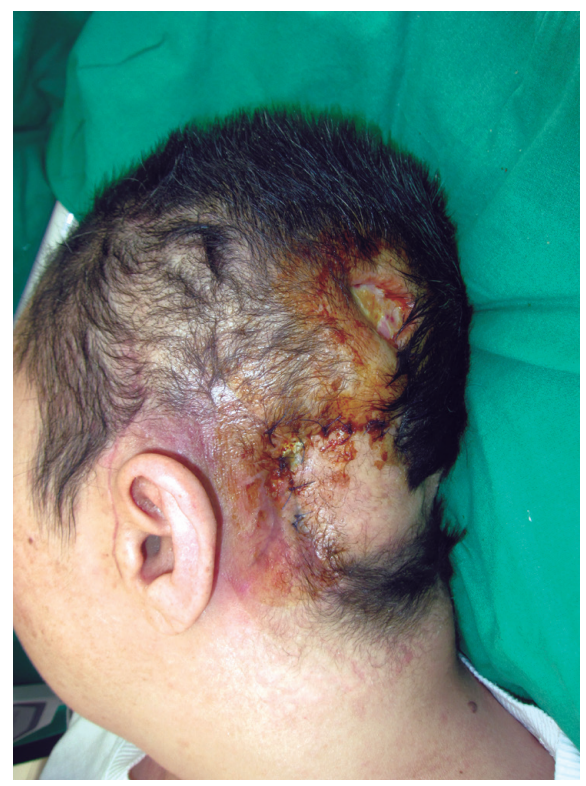

Fig. 5. Two months later, wound disruption and chronic seroma was found on previous flap site. The tumor recurrence was found.

was designed from the left occipital area and neck (Fig. 6). The flap was elevated in the suprafascial layer and rotated superiorly. To reconstruct the remaining upper portion of the defect, the previous rotation flap was re-elevated and transposed to the defect, and to cover the donor site of that flap and the previous skin graft site, an

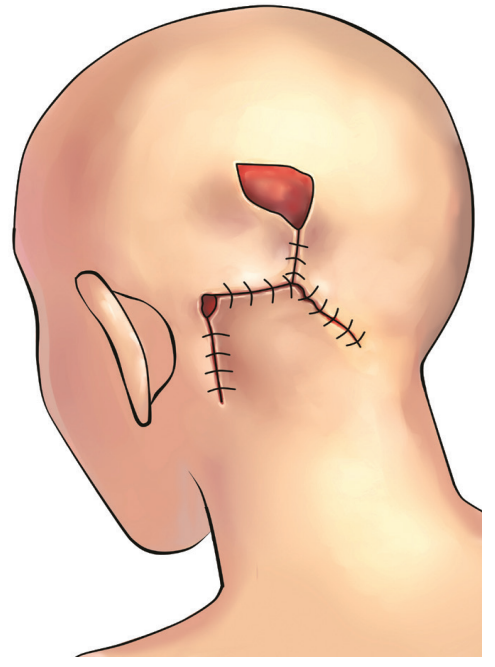

Fig. 4. Schematic illustration of harvesting rotation flap and skin grafting for donor site.

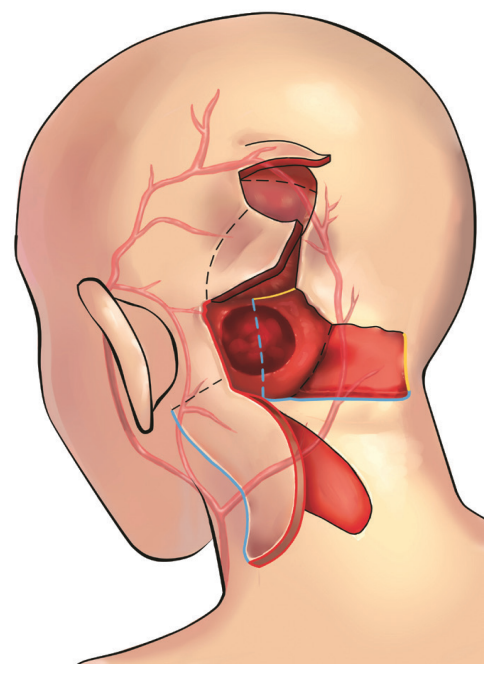

Fig. 6. Multiple perforator based island flap and rotated flap was planned. Schematic illustration shows the flap and the arterial pedicle.

additional rotation flap was designed from the vertex and transposed inferiorly (Figs. 7, 8). All donor sites were closed primarily. The patient was discharged two weeks after surgery without any complications. Two months later, the patient died. 


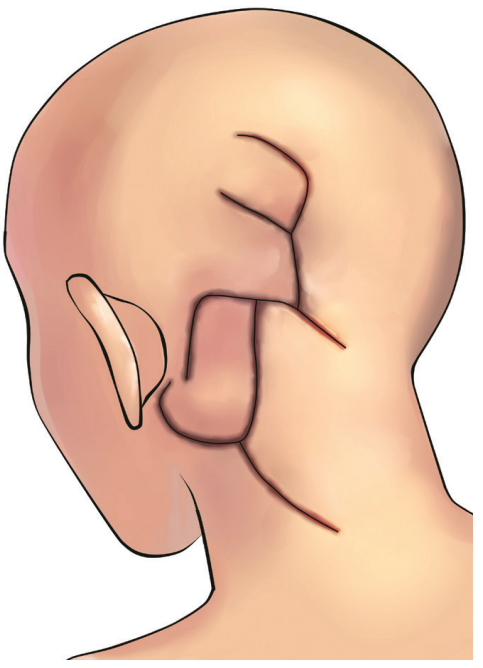

Fig. 7. Immediate postoperative schematic illustration.

\section{DISCUSSION}

Defects of the scalp can be challenging because of inelasticity of the structure [2]. Usually only small defects with wide undermining can be closed primarily, and moderate to large defects require additional surgical procedures such as skin grafts or local flaps $[1,3]$. Microvascular tissue transfer is the gold standard where the defect is extensive, there is a concomitant cranial defect, a tumor has been excised or there are traumatic avulsions or vascular malformations [2].

Microvascular tissue transfer has the advantage of being able to cover a large wound with highly vascularized tissue in a one-stage procedure [2]. A variety of free flaps have been reported; these include latissimlus dorsi, anterolateral thigh, radial forearm, serratus anterior, free omentum, rectus abdominis, and scapula flaps [1,2,7-10].

Although non-oncologic defects can be reconstructed by expanding adjacent tissue, reconstruction after oncologic resection requires immediate reconstruction without delaying treatments [6]. The critical decision is when to use free tissue transfer. The use of larger local flaps is considered the standard treatment for defects

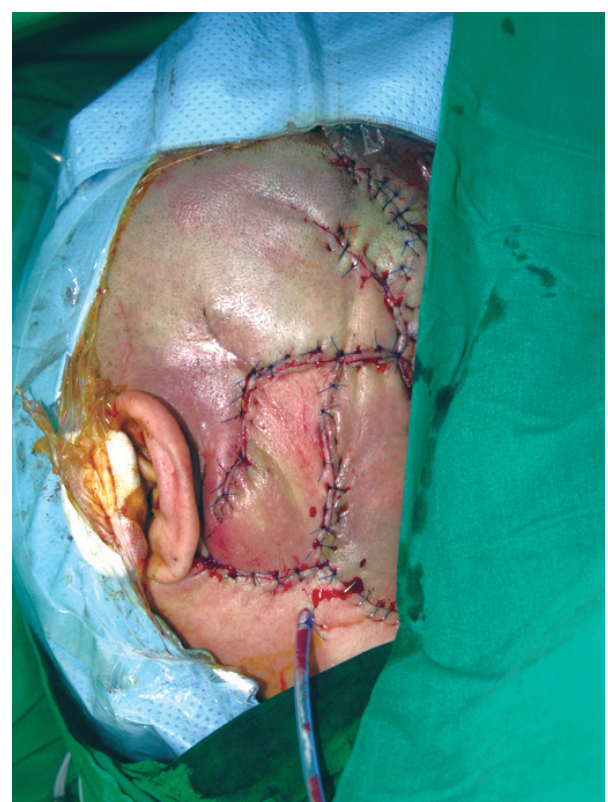

Fig. 8. A clinical photo shows nice contour and obliteration of dead space. Donor site was closed primarily.

of up to 6 to $8 \mathrm{~cm}$ in diameter. Since a local flap allows preservation of hair-bearing scalp with minimal donor site defects and excellent wound healing, it should be considered first. On the other hand, frontal defects and defects around the hairline require free tissue transfer for defects up to 4 to $5 \mathrm{~cm}$ to prevent distortion of the frontal hairline and eyebrow [6]. Especially when neo-adjuvant or adjuvant radiation therapy is used, muscle-containing free flaps reduce the risk of wound dehiscence caused by radiation injury [1,2]. Also, by using a muscle flap with a split-thickness skin graft, smooth and stable soft-tissue coverage can be achieved with a similar aesthetic outcome to that obtained with a local skin flap [6].

We also considered the muscle flap to be the ideal reconstructive modality for our patient, since immediate reconstruction was essential to cover the defect and adjuvant radiation therapy to be considered afterwards. We used a serratus anterior muscle flap with a split-thickness skin graft. The serratus anterior muscle flap can be harvested easily and quickly, which was useful for our patient who had already undergone a long operation involving radical tumor resection by neurosurgery; it also has the advantage of minimal donor site morbidity and a long vascular pedicle. After failure of the free flap, we reconstructed the defect with a bilateral 
rotational flap since the patient was unable to tolerate another long operation and quick, stable reconstruction was needed. During the primary reconstruction, including the free flap and the rotation flap after failure of the free flap, one thing we overlooked was radical surgical resection of the tumor, which was the single most important curative option [6]. Although we did not expect or plan for recurrence of the tumor, if there was a possibility of recurrence, the use of a microvascular free flap was suboptimal because the patient would require multiple repeated tumor resections following reconstruction $[6,7]$.

When recurrence of a tumor after reconstruction is suspected, additional tumor resection and reconstruction should be planned. In that situation, the reconstructive goal is to close the defect and create a stable wound. Cosmetic factors are not an issue. Although radical surgical resection is still the goal, palliative treatment should be considered due to repeated recurrence of the tumor. Therefore, in our case we used multiple local flaps because the patient was unlikely to tolerate a long surgical procedure, even though ideally the defect would be reconstructed with another microvascular free flap. With multiple local flaps, the defect was closed in a single step without skin grafting, and the patient was able to recover fast and proceed to further treatment. The microvascular free flap remained as a final option for reconstruction if the tumor was completely cured.

Even in a palliative setting, local tumor control by radical resection seems beneficial, because it significantly increase quality of life and facilitates further patient care [6]. Although hospitalization is necessary and the treatment entails risk, we believe local tumor control improves patient comfort by reducing tumor bleeding and pain, and facilitating care of the wound.

We describe a case involving repeated recurrence of a tumor following reconstruction. Although radical resection of the tumor and reconstruction seemed to have been achieved, repeated recurrences of the tumor made us to establish another reconstruction plan for the defect after oncologic surgery. The local flap was more suitable for the patient than free flap, since we could not exclude re-recurrence. In case of making a reconstruction plan following oncologic surgery, the possibility of recurrence should be taken into account, and it will reduce the patient's suffering from postoperative morbidity and complications during the rest of life.

\section{REFERENCES}

1. Sittitavornwong S, Morlandt AB. Reconstruction of the scalp, calvarium, and frontal sinus. Oral Maxillofac Surg Clin North Am 2013;25: 105-29.

2. Frodel JL Jr, Ahlstrom K. Reconstruction of complex scalp defects: the "Banana Peel" revisited. Arch Facial Plast Surg 2004;6:54-60.

3. Gundeslioglu AO, Selimoglu MN, Doldurucu T, Bekerecioglu M. Reconstruction of large anterior scalp defects using advancement flaps. J Craniofac Surg 2012;23:1766-9.

4. Hoffman JF. Management of scalp defects. Otolaryngol Clin North Am 2001;34:571-82.

5. Lesavoy MA, Dubrow TJ, Schwartz RJ, Wackym PA, Eisenhauer DM, McGuire M. Management of large scalp defects with local pedicle flaps. Plast Reconstr Surg 1993;91:783-90.

6. Iblher N, Ziegler MC, Penna V, Eisenhardt SU, Stark GB, Bannasch H. An algorithm for oncologic scalp reconstruction. Plast Reconstr Surg 2010;126:450-9.

7. Hussussian CJ, Reece GP. Microsurgical scalp reconstruction in the patient with cancer. Plast Reconstr Surg 2002;109:1828-34.

8. Disa JJ, Pusic AL, Hidalgo DH, Cordeiro PG. Simplifying microvascular head and neck reconstruction: a rational approach to donor site selection. Ann Plast Surg 2001;47:385-9.

9. Chang DW, Langstein HN, Gupta A, De Monte F, Do KA, Wang X, et al. Reconstructive management of cranial base defects after tumor ablation. Plast Reconstr Surg 2001;107:1346-55.

10. Lutz BS, Wei FC, Chen HC, Lin CH, Wei CY. Reconstruction of scalp defects with free flaps in 30 cases. Br J Plast Surg 1998;51:186-90. 